\title{
1 Toward the Control of Self-Assembling Systems
}

\author{
Eric Klavins ${ }^{\star}$ \\ California Institute of Technology, Pasadena, CA 91125, USA
}

\begin{abstract}
In recent work [4], capillary forces between tiles floating on a liquidliquid interface are used to direct a self-assembly process. By carefully arranging the wetabilities of the edges of the tiles, regular arrays of various shapes spontaneously form when the tiles are gently shaken. It is difficult, however, to avoid flaws in the assembled aggregates and to assemble terminating and asymmetric structures. In this paper, we suppose that the wetability properties of the tiles, and therefore the capillary forces, can be controlled. In particular, we introduce a simple model of a "waterbug" shaped tile and derive the equations of motion for a system of such tiles from a model of the lateral forces between two floating colloidal particles. We then explore the possibilities for control in this setting and present some initial forays into addressing the above difficulties.
\end{abstract}

\section{$1.1 \quad$ Introduction}

Self-assembly plays a crucial role in many phenomena in chemistry, physics and cell biology. Self assembling systems occur when many similar parts (molecules, colloids, tiles) are placed in an environment that thermodynamically favors their forming regular arrays. Recent work on mesoscale selfassembly [4] has employed capillary forces between millimeter scale tiles floating on a liquid-liquid interface to direct the assembly process. By carefully arranging the wetabilities of the edges of the tiles, regular arrays of various shapes spontaneously form as the system is gently shaken. The straightforward construction of such tile systems allows researchers to more easily observe the assembly process than in chemical systems. Mesoscale self-assembly may also have practical applications such as the assembly of three dimensional memory chips [5] or of computer displays [24].

Two main difficulties arise in self-assembling systems. First, it is difficult to avoid flaws in the assembled aggregates due to malformed parts or, more interestingly, local minima in the energy landscape. Second, it can be difficult to assemble terminating and asymmetric structures for arbitrary part morphologies. For example, square parts with all hydrophobic edges form square lattices that terminate only when all parts are incorporated. Designing an open loop system that terminates at, for example, multiple $10 \times 10$ arrays of such parts is not possible.

\footnotetext{
* This research is supported in part by DARPA grant number F33615-98-C-3613 and by AFOSR grant number F49620-01-1-0361.
} 


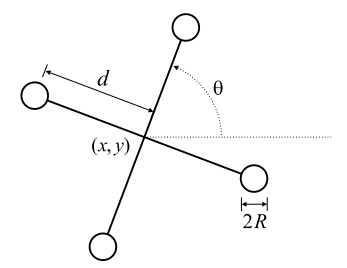

Fig. 1.1. The waterbug tile model: Two lightweight beams of equal length joined at their centers at right angles. To the ends of each beam are attached buoyant particles whose wetability (see Figure 1.3) can be controlled. When the tile floats on the surface of the water, the meniscus formed by the "feet" results in attraction to or repulsion from the feet of other tiles.

One way to address these problems is to suppose that the parts themselves have some control over in which binding interactions they participate. In a biochemical environment, we might suppose that this control is mediated by organelles in the cell. In mesoscale self-assembly, we might suppose that tiles have control over the wetabilities of their edges and also some idea of their local state.

Of course, other models of self-actuated, robotic parts using magnets or latches are readily imagined. In this paper, however, we propose a model of a tile, dubbed the waterbug (Figure 1.1), we believe will be useful in developing an initial analytical understanding of the dynamics and control of self-assembling systems. The waterbug consists of two beams held at right angles with buoyant particles (its feet) at the ends of the beams. We suppose that the wetabilities of the feet can be varied $[22,13]$ so that their interactions with other feet can be controlled. This model is conceptually simple in that we can, using what is known about particles floating on liquid-fluid interfaces (Section 1.3.1 which summarizes [16]), derive fairly accurate equations to describe its motion and its interaction with other tiles (Section 1.3.2).

Remark: We emphasize that the details of the waterbug model are not important. The forces that attract particles together could equally well be magnetic, electrostatic, even gravitational, and the qualitative behavior of the system would be the same: The tiles readily form aggregates that are, qualitatively, quite similar to those observed in [4] as illustrated in Figure 1.2 .

The opportunities for new modeling and control techniques in self-assembly are substantial. In general, the main difficulties are the estimation of global state from local information and distributed control based on local and incomplete information. After introducing the waterbug model, we suggest some initial ideas for how to address these difficulties. In Sections 1.3.3 and 1.3.4, we examine the dynamics of the assembly model we propose and our initial understanding of what kinds of aggregates are possible. In Section 1.4.1, we demonstrate that by controlling the wetabilities of the feet of the waterbug 


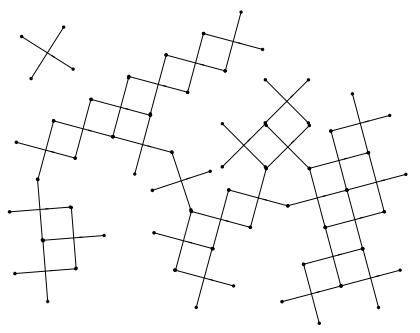

Fig. 1.2. An example intermediate assembly of waterbug tiles. Without control, the tiles arrange themselves into quasi-regular arrays that terminate only when all tiles are incorporated. Defects, corresponding to undesirable stable configurations (see for example Figure 1.6(c)), are also possible.

tile, we can avoid certain undesirable subassemblies that would have been energetically favorable if the wetabilities of the feet were static. In Section 1.4.2 we review $[9,10]$ and suggest how the methods used there can be adapted to the present model.

\subsection{Related Work}

Research in self-assembly has traditionally been the realm of supramolecular chemistry [14] wherein the formation of regular molecular aggregates by noncovalent binding interactions (such as hydrogen bonding) is studied. Such aggregates form spontaneously due to thermodynamics and chance collisions and are not mediated by chemical reactions. Many examples of these aggregates and their assembly dynamics, from dendrimer formation to chemical contaminant recognition, can be found in the recent issue of the PNAS dedicated to the subject [8]. Molecular self-assembly systems are quite flexible and in fact, in $[2,23]$, arbitrary computations are studied using DNA tiles, reinforcing the view put forth in [14] that self-assembly is as much about structure as it is about information.

Research into aggregates of small particles suggests alternative systems for which interesting self-assembling systems can be easily constructed and observed. According to [6], Perrin was the first (in 1909) to report the phenomenon wherein particles in fluid are attracted to each other by capillary forces. The idea is extended significantly in [4] to systems of tiles with various shapes floating on a liquid-liquid interface. By carefully designing the wetabilities of the edges of the tiles with respect to one of the liquids, a wide variety of arrays of tiles can be made. This work was further extended [20] to reproduce, with tiles and capillary forces, the computational systems investigated in [23]. Chemistry and colloid physics are combined in [15] wherein single strands of DNA are attached to small gold balls which then assemble according to the interactions between complementary strands of DNA. 
Saitou [21] has explored the logic of conformational switching whereing the binding of a part to a subassembly changes the "shape" and therefore the future binding properties of the parts involved.

The idea of controlling the wetability of surfaces is used in [13] to move a liquid droplet across the surface of a microchip (see also [1]) and in [22] for protein patterning. More generally, capillary forces are being used to a greater and greater extent in the MEMs community for microfluidic manipulation and assembly [7].

In robotics and control, Koditschek was the first (in unrelated work) to propose the idea of assembly as a "game of its pieces" [19]. In this work, parts can move themselves - or they can direct external manipulators to move them - based on artificial potential functions [12]. The robotics community, however, has mainly latched onto this work for its application to robot navigation. Initial forays have also been made toward decentralized versions of this idea [18] that resemble self-assembling systems, but for which analysis is apparently quite difficult.

Despite the innovation of considering parts as autonomous, the approach in [19] is problematic in that the programs that achieve a final desired configuration (assembly) are global. We are concerned with systems where global information is not readily available if for no other reason than that the size of the system is enormous, so that the communication complexity of sharing global information [11] is prohibitively high. To overcome this we have examined decentralized schemes for assembly $[9,10]$, which require only local knowledge and which, statistically, seem to work. The main idea is to compile programs for each autonomous part from a specification of the desired final assembly, which in general may be terminating and asymmetric - unlike most uncontrolled tile systems [4]. When the programs are run by each part, copies of the desired assembly form. The method relies on local sensing and control: We augment the natural thermodynamic programs of the tiles with internal state and control. The work in $[9,10]$ assumes a certain highly idealized part. One motivation for the the present work is to investigate the feasibility of

various physically realistic models for autonomous parts that may be able to execute the programs compiled in $[9,10]$.

\subsection{Modeling}

\subsubsection{Lateral Capillary Forces Between Floating Particles}

Consider the system depicted in Figure 1.3. Two particles float on a liquidfluid interface (e.g. water-air). Each particle $k$ forms a meniscus which has slope $\Psi_{k}$ based on the wetability of the particle. If the particle is hydrophilic, then $\Psi_{k}>0$. Otherwise the particle is hydrophobic and $\Psi_{k}<0$. The two particles will experience a force along the line connecting them due to the thermodynamic tendency for the interfacial free energy between the liquid and fluid to be minimized. Using the Laplace-Young [17] equation, which 


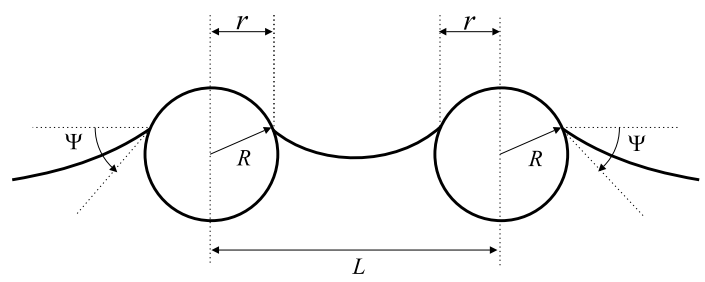

Fig. 1.3. The model examined in [16]: Two particles floating on a liquid-fluid interface form a meniscus that results in their mutual attraction or repulsion, depending on their respective wetabilities. The waterbug model in Figure 1.1 uses such particles for its "feet".

describes the shape of the meniscus around the particles, Paunov et al. [16] determined that the lateral force between two particles at distance $L$ from each other is approximated by

$$
F(L)=2 \pi \gamma Q_{1} Q_{2} \rho K_{1}(\rho L)\left[1+\mathrm{O}\left(\rho^{2} R^{2}\right)\right]
$$

where $\gamma$ is a parameter describing the surface tension of the interface; $\rho$ is a parameter (related to $\gamma$, the mass densities of the liquid and fluid, and the force of gravity) describing the capillary length; $R$ is the radius of particles; $K_{1}$ is the modified Bessel function of the first order; and

$$
Q_{k} \triangleq r_{k} \sin \Psi_{k}
$$

where $r_{k}$ is the distance from the center-line of the particle to the interface (see Figure 1.3). We will assume that $\rho^{2} R^{2} \ll 1$ so that this approximation is valid. However, we will be noncommittal about the rest of these parameters in this paper.

Using the fact that $K_{1}(\rho L)>0$ and the definition of $Q_{k}$, we see that two particles of similar wetabilities (both hydrophobic, or both hydrophilic) will be attracted to each other, whereas two particles of differing wetabilities will be repelled from each other.

To simplify notation, define $c_{1,2} \triangleq 2 \pi \gamma Q_{1} Q_{2}$. Using the fact that

$$
\frac{\partial}{\partial x} K_{0}(x)=-K_{1}(x)
$$

where $K_{0}$ is the modified Bessel function of zeroth order, we see that (1.1) is a gradient field with potential energy function

$$
U_{\text {pre }}(L)=-c_{1,2} K_{0}(\rho L) .
$$

This function is depicted (with two variations of it) in Figure 1.4. Note in particular that $U_{\text {pre }}(L)$ is singular at $L=0$. 


\subsubsection{The Waterbug Model}

We are in general interested in systems of tiles, such as those examined in [4], whose edges have certain wetability properties. To understand such systems using what we know about particle interactions, we introduce a model "waterbug" tile (shown in Figure 1.1) that is similar to the tiles studied experimentally in, for example, [4], with respect to its possible interactions with other tiles. The waterbug model consists of two beams of negligible mass and of length $2 d$ joined at their centers at right angles. To the ends of each beam are attached buoyant particles (which we will call feet) of radius $R$, mass $m$ and with wetabilities described by $\Psi_{1}, \Psi_{2}, \Psi_{3}$ and $\Psi_{4}$.

We suppose that the $\Psi_{j}$ are control inputs to the system; that is, we suppose that a tile is able, somehow $[13,1,22]$, to vary the wetabilities of its feet so that they will be attracted to or repelled by the feet of other tiles according to (1.1).

Now consider a system consisting of $n$ waterbug tiles. Denote by $q_{i}=$ $\left(x_{i}, y_{i}, \theta_{i}\right)$ the generalized position and orientation of the $i$ th tile and by $w_{i, j}=\left(u_{i, j}, v_{i, j}\right)$ the actual position of the $j$ th foot of tile $i$ (e.g. $\left(u_{i, 1}, v_{i, 1}\right)=$ $\left.\left(x_{i}+d \cos \theta_{i}, y_{i}+d \sin \theta_{i}\right)\right)$. We denote the full state of the system $\left(q_{1}, \ldots, q_{n}\right)$ by q. The potential energy corresponding to two different tiles $i$ and $k$ is

$$
U_{\text {tiles }}\left(q_{i}, q_{k}\right)=-\sum_{j=1}^{4} \sum_{l=1}^{4} c_{i, j, k, l} K_{0}\left(q\left\|w_{i, j}-w_{k, l}\right\|\right)
$$

where $c_{i, j, k, l}=2 \pi \gamma Q_{i, j} Q_{k, l}$ and $Q_{i, j}$ is the wetability coefficient of the $j$ th foot of the $i$ th tile. We have assumed that no two feet are touching (i.e. that $\left\|w_{i, j}-w_{k, l}\right\|>2 R$ for all $i, j, k$ and $\left.l\right)$. The full potential energy of the system $^{1}$ is then

$$
U(\mathbf{q})=\sum_{1 \leq i \neq k \leq n} U_{w}\left(q_{i}, q_{k}\right) .
$$

The kinetic energy of a single tile is $K_{i}=4 m\left(\dot{x}_{i}+\dot{y}_{i}+d^{2} \dot{\theta}_{i}\right)$, and of the system is $K=\sum_{i=1}^{n} K_{i}$. We assume that each foot is subject to the force of viscous friction which we model simply by $-k_{f} \dot{w}_{i, j}$ where $k_{f}>0$ is a constant. Setting $L=K-U$ and setting the Lagrangian equal to the sum of the frictional forces, we have that the dynamics of the system are described by

$$
\begin{aligned}
8 m \ddot{x}_{i}+\sum_{k=1, k \neq i}^{n} \frac{\partial}{\partial x_{i}} U_{w}\left(q_{i}, q_{k}\right) & =-4 k_{f} \dot{x}_{i} \\
8 m \ddot{y}_{i}+\sum_{k=1, k \neq i}^{n} \frac{\partial}{\partial y_{i}} U_{w}\left(q_{i}, q_{k}\right) & =-4 k_{f} \dot{y}_{i} \\
8 m d^{2} \ddot{\theta}_{i}+\sum_{k=1, k \neq i}^{n} \frac{\partial}{\partial \theta_{i}} U_{w}\left(q_{i}, q_{k}\right) & =-4 k_{f} d^{2} \dot{x}_{i} .
\end{aligned}
$$




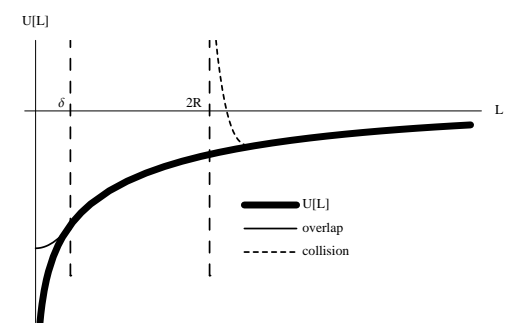

Fig. 1.4. The potential energy $U_{\text {pre }}(L)$ between two particles distance $L$ apart and the modified potentials used to model contact situations. The collision potential, if used, is active when $L<2 R$. The smoothing of the singularity, if used, is active when $L<\delta \ll R$. In both cases a polynomial of appropriate degree is used so that the resulting potential is $C^{2}$.

\subsubsection{The Hybrid Dynamics of Assembly Systems}

The equations (1.4) are valid under the assumption that $\left\|w_{i, j}-w_{k, l}\right\|>2 R$ for all $i, j, k$ and $l$. When two feet make contact, however, the system dynamics change. There are three obvious ways to describe the system wherein some number of feet are touching.

First, we can define a hybrid automaton [3] with states corresponding to each of the $4 n(4 n-4)$ possible contact configurations. In each state the dynamics are described by (1.4) subject to the constraints $\left\|w_{i, j}-w_{k, l}\right\|=2 R$ for each pair $((i, j),(k, l))$ of contacting feet. Transitions occur between states when new pairs come into contact or when the force (1.1) holding two feet together is overcome by other forces. This model is appealing because the finite state part mirrors nicely the space of all possible assembly sequences. It is unappealing for analytical and simulation purposes because of possible chattering between states and the neutral stability of equilibria due to the fact that $\left\|w_{i, j}-w_{k, l}\right\|=2 R$ defines a circle and not a point.

Second, we can modify the potential function $U_{\text {pre }}$ in (1.3) so that a (large) force preventing two particles from overlapping takes affect when $\| w_{i, j}-$ $w_{k, l} \|<2 R+\delta$ as in Figure 1.4. This is very appealing for simulation purposes as it eliminates chatter and is fairly realistic: It has been observed [4] that a thin layer of liquid separates two tiles that are essentially in contact. However, the analysis of stable configurations (possible final aggregate shapes) (Section 1.3.4) is difficult with this model due to the piecewise and ad hoc nature of the modified potential.

Third, we can suppose that two feet can overlap (occupy the same place). If $R \ll d$, then two feet in contact is essentially the same as two feet in the same place. For purposes of simulation, we smooth out the singularity in $U_{\text {pre }}$

\footnotetext{
${ }^{1}$ We assume that the potentials are simply additive as, for example, with particle systems under the influence of gravity. It is not known whether this assumption can be shown from first principles from the Laplace-Young Equation.
} 
with an appropriate polynomial potential that is in effect when $\left\|w_{i, j}-w_{k, l}\right\|<$ $\delta \ll 2 R$ (see Figure 1.4). In this manner we are able to numerically simulate systems of up to 40 tiles in a few hours. Further supposing that $U_{\text {tiles }}$ is in effect only when tiles are "close by" results in efficient simulation of even larger systems.

For analytical purposes, we proceed much as in the first case and consider a system of waterbug tiles linked by certain feet to form a two dimensional mechanical linkage of tiles (as in Figures 1.5 and 1.6). In particular, let $V=\{1, \ldots, n\} \times\{1, \ldots, 4\}$ correspond to the set of all $4 n$ feet and let $E$ be an equivalence relation over $V$ that specifies which feet are collocated. The submanifold corresponding to the constraints imposed by $E$ is

$$
M_{E} \triangleq\left\{\mathbf{q}:[(i, j),(k, l)] \in E \Rightarrow w_{i, j}\left(q_{i}\right)=w_{k, l}\left(q_{k}\right)\right\} .
$$

(It may be that $M_{E}=\emptyset$ ). The equations of motion are then similar to (1.4) except projected onto $M_{E}$. To obtain them, however, we can not use $U(\mathbf{q})$ since it is singular if $E$ is nontrivial. Thus, define the set of non-collocated feet by

$$
\mathcal{F}_{E} \triangleq\{(i, j, k, l):[(i, j),(k, l)] \notin E\}
$$

and set

$$
U_{E}(\mathbf{q}) \triangleq-\sum_{(i, j, k, l) \in \mathcal{F}_{E}} c_{i, j, k, l} K_{0}\left(q\left\|w_{i, j}\left(q_{i}\right)-w_{k, l}\left(q_{k}\right)\right\|\right) .
$$

The equations of motion of the partially assembled system given by $E$ are then

$$
\frac{d}{d t}\left(\frac{\partial\left(K-U_{E}\right)}{\partial \dot{\mathbf{q}}}\right)-\left(\frac{\partial\left(K-U_{E}\right)}{\partial \mathbf{q}}\right)=F_{\text {friction }} .
$$

subject to the constraints

$$
[(i, j),(k, l)] \in E \Rightarrow w_{i, j}\left(q_{i}\right)=w_{k, l}\left(q_{k}\right)
$$

for all $i, j, k$ and $l$.

\subsubsection{Stable Assemblies}

Given an assembly specified by $E$, any configuration $\mathbf{q}^{*}$ for which $-\nabla U_{E}\left(\mathbf{q}^{*}\right)$ is normal to $M_{E}$ at $\mathbf{q}^{*}$ is an equilibrium of (1.5) subject to (1.6). We have:

Proposition 1. If all points in $M_{E}$ are equilibria of (1.5) subject to (1.6), then the assembly given by $E$ is stable (modulo rotation and translation). 


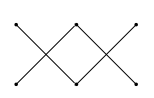

(a)

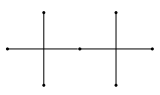

(b)

Fig. 1.5. The two possible assembled configurations of two waterbug tiles (up to symmetry and excluding overlapping crossbars). (a) is stable, whereas (b) is unstable but converges to (a).

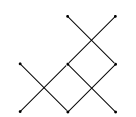

(a)

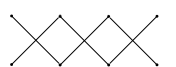

(b)

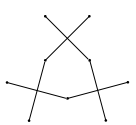

(c)

Fig. 1.6. The three possible stable assembled configurations of three waterbug tiles (up to symmetry and excluding overlapping crossbars). In similar tile systems, configurations like (a) and (b) are desirable, while (c) may not be: If a system with a larger number of tiles were "seeded" with (c), irregular aggregates with holes would form (as in Figure 1.2).

In particular, for all $\Upsilon_{i, j}$ positive, the criterion in Proposition 1 is simply another way of stating that the mechanical linkage described by $E$ is in fact rigid. Non-rigid assemblies can have unstable at equilibrium points. For example, the assemblies in Figures 1.5(a) and 1.6(a)-(c) are stable. The assembly in Figure 1.5(b) is an unstable equilibrium (with respect to $\theta_{1}-\theta_{2}$ ).

It is also possible that an equilibrium of a non-stable assembly is itself stable. In our simulations, such configurations seem to occur exclusively when the beams of two different tiles overlap - although this remains to be confirmed.

Given an assembly specified by $E$, the system described by (1.5) and (1.6) will have singularities at any point $\mathbf{q}^{*} \in M_{E}$ such that, for some $i, j, k$, and $l, w_{i, j}\left(q_{i}^{*}\right)=w_{k, l}\left(q_{k}^{*}\right)$ since

$$
\lim _{L \rightarrow 0} U_{p}(L)=-\infty .
$$

Thus, at such a point, the equations of motion are not locally Lipschitz and solution trajectories are not guaranteed to be unique. Nevertheless, such configurations can be shown to be locally attracting using a variation of Lyapunov's direct method. For example, configuration 1.5(b) has two attracting configurations isomorphic to $1.5(\mathrm{a})$.

In [4], the observation is made that some configurations are less stable than others. They use this property to their advantage by adding enough energy to the system (by gently shaking it) so that undesirable assemblies are energetically unfavorable while desirable ones are. We have not yet developed analytical means for determining the energy required to break up various classes of waterbug assemblies - although we have observed in our simulations 
that, for example, the assembly in Figure 1.6(c) is less stable (in the presence of disturbances resulting from collisions with other tiles and assemblies) than the assemblies in Figures 1.6(a) or (b).

A complete catalog of possible waterbug assemblies is not known. More generally, given a tile morphology, an automatic method for determining the stable assemblies of a soup of such tiles (and their energies) is not known.

\subsection{Discussion}

The essential control task for an assembly system, in its most basic form, is to bring the system from an initial assembly $E_{0}$ to a final assembly $E_{N}$ that is one of some prespecified family of final (stable) assemblies $\mathcal{E}_{\text {final }}$. Thus, we desire a sequence $E_{0}, \ldots, E_{N}$ where $E_{0}$ is the identity relation, $E_{N} \in \mathcal{E}_{\text {final }}$ for any $t, E_{t} \subseteq E_{t+1}$. Furthermore, there may be some set $\mathcal{B}$ of undesirable configurations (an obstacle in a sense) so that we require that $E_{t} \notin \mathcal{B}$ for any $t \in\{0, \ldots, N\}$ (see Figure 1.7).

We have so far supposed that the actuation model for these systems is the variable wetabilities of the tile feet. Of course, less direct (and possibly more practical) actuation models exist (such as shaking the system and thereby keeping its energy at a certain level unfavorable to the formation of assemblies in $\mathcal{B}$ ). For the rest of this section though, we assume the first sort of actuation. Of the many control tasks one might imagine for these self-assembling systems, we briefly consider two: eliminating defects and obtaining terminating structures.

\subsubsection{Eliminating Defects}

Figure 1.2 shows an intermediate assembly containing the (possibly undesirable) sub-configuration shown in Figure 1.6(c). For a system of three tiles, the domain of attraction of the configuration in Figure 1.6(c) is small but nevertheless measurable. For example, for tiles with all-like wetabilities, the initial configuration in Figure 1.7(a) leads to Figure 1.6(c). However, an open loop controller defined by

$$
\Psi_{i, 2}(t)=\Psi_{i, 4}(t)=\left\{\begin{array}{l}
0 \text { if } t<t_{1} \\
\Psi \text { otherwise }
\end{array}\right.
$$

and $\Psi_{i, 1}(t)=\Psi_{i, 3}(t)=\Psi$ for all $i \in\{1,2,3\}$ where $\Psi>0$ is a constant, results in a system that avoids Figure 1.6(c). Essentially, with $t<t_{1}$, Figure 1.6(c) is not an attracting configuration. Thus, with the wetabilities arranged as they are with $t<t_{1}$, the configuration in Figure 1.7(c) is obtained. Once this is arrived at, there is no (energetically decreasing) way to get to 1.6(c) and therefore, with all wetabilities positive (for $t \geq t_{1}$ ), Figure 1.7(e) is obtained. 


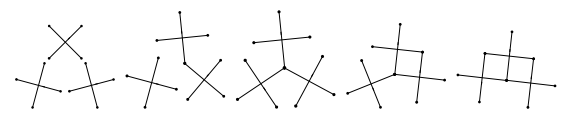

(a)

(c)

(d)

(e)

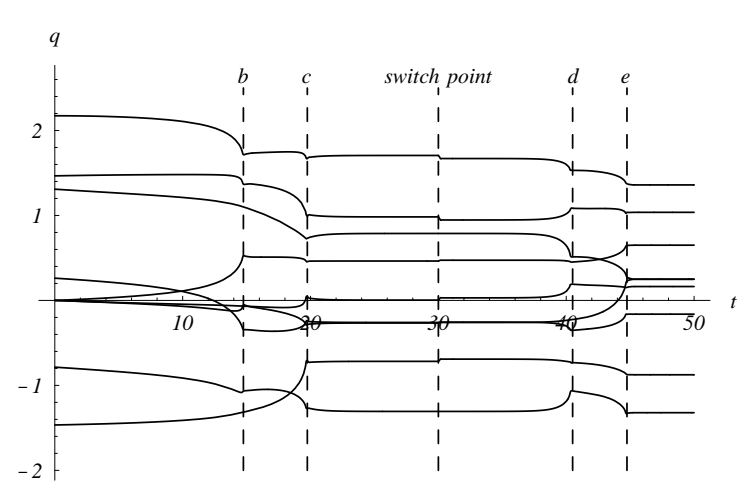

Fig. 1.7. Trajectory $\left(x_{1}(t), y_{1}(t), \ldots\right)$ of a three tile system with initial conditions (a) very close to $1.6(\mathrm{c})$, controlled to avoid this configuration. From time $t=0$ to time $t=30$, feet $\Psi_{i, 2}=\Psi_{i, 4}=0$ and $\Psi_{i, 1}>0$ and $\Psi_{i, 3}>0$ for $i \in\{1,2,3\}$. For $t>30$, all $\Psi_{i, j}>0$ for all $i$ and $j$. Times (b)-(d) show intermediate configurations.

\subsubsection{Terminating Structures}

In general a given (uncontrolled) tile shape will result in a certain family of structures that can be assembled. This family is the closure of the operation of adding a new part to a given assembly, if possible. The waterbug tile with all hydrophobic feet has an infinite family of assemblies: a new tile can always be added onto any stable assembly to make a new stable assembly. The problem of generating terminating structures is to add control algorithms to the tiles so that the assembly family formed consists only of the desired finite structures and their subassemblies (as, for example, in Figure 1.8).

In $[9,10]$ we describe a method for assembling such terminating structures out of 2D, self-actuated disk-shaped parts. In particular, any tree-shaped structure (directed, acyclic graph) can be assembled and the local rules directing the assembly can be automatically and efficiently synthesized. The method is shown to be correct under certain assumptions about the assembly dynamics. It requires that each tile be able to sense the discrete state (defined below) of tiles near it and that it be able to change the wetabilities of its feet. In the rest of this section, we outline how a simple variant of this method can be used to assemble a certain tree-shaped assembly: number 6 in Figure 1.8. Although the controller described here is constructed by hand for a particular assembly, the method in $[9,10]$ can easily be adapted to automatic synthesis of controllers for any tree-shaped assembly. 

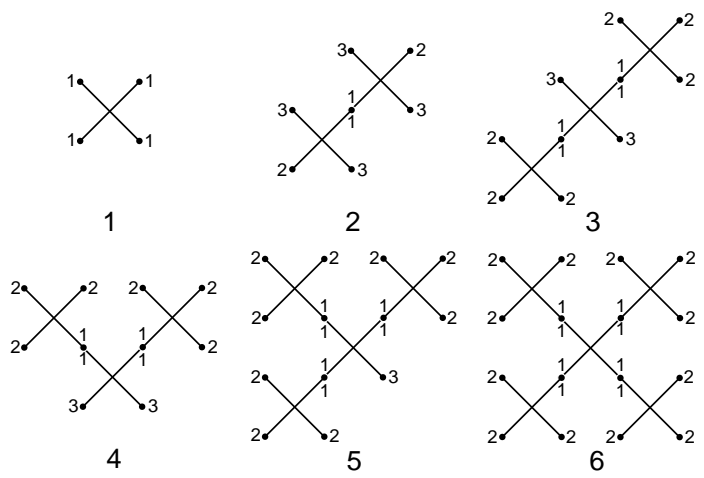

Fig. 1.8. An example terminating structure (number 6) and its subassemblies up to isomorphism. Each subassembly is given an index (1-6) and each foot of each tile is given a role identifier. Thus, each foot in a subassembly has a discrete state corresponding to its subassembly-role pair. The state is used to determine which binding interactions between pairs of tiles should occur.

A desired assembly of $n$ tiles is specified by an equivalence relation $E_{\text {spec }}$ over $1, \ldots, n \times\{1, \ldots, 4\}$ as in Section 1.3.3. The first step in generating rules that result in the assembly of copies of $E_{\text {spec }}$ is to form a list $\mathcal{E}$ of its subassemblies (including $E_{\text {spec }}$ ). For the present example, this list is shown in Figure 1.8 as subassemblies 1 through 6 . The next step is to identify the role of each foot of each tile in each subassembly. These are given by the small numbers located next to the feet in the figure.

Given $\mathcal{E}$ and an assignment of roles, the discrete state of a waterbug foot is defined to be its subassembly-role pair $(s, r)$. For example, any tile that is not joined to another tile has subassembly-role pair $(1,1)$. A foot in a twotile assembly will have subassembly-role pair $(2,1),(2,2)$ or $(2,3)$. We now construct an algebra of subassemblies.

Definition 1. Given $\left(s_{1}, r_{1}\right)$ and $\left(s_{2}, r_{2}\right)$, we define their join $\left(s_{1}, r_{1}\right) \oplus\left(s_{2}, r_{2}\right)$ to be $s$ if the action of joining subassemblies $s_{1}$ and $s_{2}$ at feet with roles $r_{1}$ and $r_{2}$ results in a subassembly isomorphic to a subassembly in $\mathcal{E}$ with index $s$. Otherwise, $\left(s_{1}, r_{1}\right) \oplus\left(s_{2}, r_{2}\right)=\perp$. If $\left(s_{1}, r_{1}\right) \oplus\left(s_{2}, r_{2}\right) \neq \perp$, then the join is called valid.

In our running example, the valid joins are given by

$$
\begin{array}{lll}
(1,1) \oplus(2,2)=3 & (1,1) \oplus(2,3)=4 & (1,1) \oplus(3,3)=5 \\
(1,1) \oplus(4,3)=5 & (1,1) \oplus(5,3)=6 . &
\end{array}
$$

Note that because of the simplicity of the example, $(1,1)$ is a part of every valid join although, in general, this is not the case.

Next we define discrete update rules for each possible join. For example, if two tiles join via feet with states $(1,1)$ and $(2,2)$, then the roles of the 


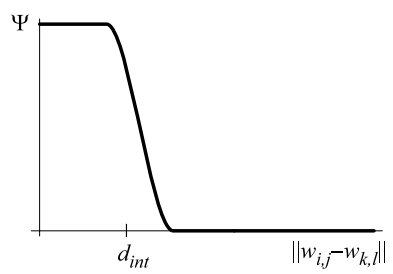

(a) Attraction assignment

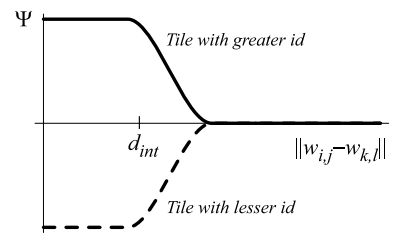

(b) Repulsion assignment

Fig. 1.9. Wetability assignments used by two waterbug tile feet as a function of their distance. If the two tiles are to attract (according to the rules described in Section 1.4.2), then they use the assignment function in (a). Otherwise they use the functions in (b) where the foot whose tile has the greater id forms a positive meniscus and the foot whose tile has the lesser id forms a negative meniscus, resulting in a repulsive force between the two feet (as described in Section 1.3.1).

subassemblies involved change according to:

$$
\begin{aligned}
(1,1),(2,2) \stackrel{(1,1) \oplus(2,2)}{\longrightarrow}\left\{\begin{array}{l}
(3,1) \text { if interacting foot } \\
(3,2) \text { else }
\end{array}\right. \\
(2,1) \stackrel{(1,1) \oplus(2,2)}{\longrightarrow}(3,1) \\
(2,3) \stackrel{(1,1) \oplus(2,2)}{\longrightarrow}\left\{\begin{array}{l}
(3,3) \text { if interacting tile } \\
(3,2) \text { else }
\end{array}\right.
\end{aligned}
$$

With the join algebra and the update rules, we can define control laws for each foot to run. We assume that the tiles are being gently shaken to encourage interactions between feet. If two feet $(i, j)$ and $(k, l)$ come in close proximity, they change their wetabilities to either attract or repel according to whether their join is valid (see Figure 1.9). If two feet join, the subassembly-role pairs of the feet in the subassemblies involved are updated according to the update rules just described. Waterbug feet may also need to repel other feet in the same subassembly to which they are not directly attached. If no interaction is desired (there are no nearby feet), the wetability assignment is set to 0 (no meniscus).

The method in $[9,10]$ also accounts for deadlock situations arising either geometrically (some tiles are blocking other tiles from useful interaction sites) or logically (for example, all subassemblies with index 1 are used up before any subassemblies of index 6 form). The deadlock mechanism is simple: If no interactions have occurred with a non-final subassembly for longer than some predetermined time, the assembly breaks up (using the repulsion assignment in Figure 1.9) and tiles formerly joined "ignore" each other (so that different interactions may occur). These rules are proved to assemble a maximum number of final assemblies, in a perfect model of interaction (i.e. different from the model described here), in [9]. 
We are presently exploring possible implementations of the assembly algorithm described above - with tiles or robots - and hope to report on our results in forthcoming publications.

\subsection{Conclusion}

We have introduced a model of a self-assembling tile system based on the physics of capillary forces. The tiles in the model are amenable to control actions via changing their wetabilities. We have demonstrated a simple open loop controller that can be used to avoid defects in the assembly process and have suggested a way to use previous work $[9,10]$ to direct the assembly process even further to build arbitrary terminating structures.

There are many other avenues and opportunities for the control of assembly systems based on different interaction and binding mechanisms, alternate actuation paradigms, and so on. We hope that the model we propose here will serve as a good starting point for such investigations.

\section{Acknowledgments}

The author would like to thank Richard Murray, Dan Koditschek and Karl Böhringer for their advice and suggestions regarding this paper and my research in self-assembly in general.

\section{References}

1. N. L. Abbott, C. B. Gorman, and G. M. Whitesides. Active control of wetting using applied electrical potentials and self-assembled monolayers. Langmuir, 11(1):16-18, 1995.

2. L. Adleman. Molecular computation of solutions to combinatorial problems. Science, 266:1021-1024, 1194.

3. R. Alur, C. Courcoubetis, T. A. Henzinger, and P.-H. Ho. Hybrid automata: An algorithmic approach to the specification and verification of hybrid systems. In Hybrid Systems I, volume 763 of LNCS, pages 209-229. Springer, 1993.

4. N. Bowden, A. Terfort, J. Carbeck, and G. M. Whitesides. Self-assembly of mesoscale objects into ordered two-dimensional arrays. Science, 276(11):233235, April 1997.

5. T. L. Breen, J. Tien, S. R. J. Oliver, T. Hadzic, and G. M. Whitesides. Design and self-assembly of open, regular, 3D mesostructures. Science, 284:948-951, 1999.

6. N. D. Denkov, O. D. Velev, P. A. Kralchevsky, I. B. Ivanov, H. Yoshimura, and K. Nagayama. Mechanism of formation of two-dimensional crystals from latex particles on substrates. Langmuir, 8:3183-3190, 1992.

7. A. Greiner, J. Lienemann, J. G. Korvink, X. Xiong, Y. Hanein, and K. F. Böhringer. Capillary forces in micro-fluidic self-assembly. In Fifth International Conference on Modeling and Simulation of Microsystems (MSM'02), pages 2225, April 2002. 
8. J. Halpern, editor. Proceedings of the National Academy of Sciences: Special Issue on Supramolecular Chemistry and Self-Assembly, volume 99:8. National Academy of Sciences, April 2002.

9. E. Klavins. Automatically synthesized controllers for distributed assembly: Partial correctness. In Proceedings of the Conference on Cooperative Control and Optimization. Kluwer, Gainsville, FL, November 2001.

10. E. Klavins. Automatic synthesis of controllers for assembly and formation forming. In International Conference on Robotics and Automation, Washington DC, May 2002.

11. E. Klavins. Communication complexity of multi-robot systems. In Workshop on the Algorithmic Foundations of Robotics, December 2002. Submitted for review.

12. D.E. Koditschek. An approach to autonomous robot assembly. Robotica, 12:137-155, 1994.

13. J. Lee and C.-J. Kim. Surface-tension-driven microactuation based on continuous electrowetting. Journal of Microelectricalmechanical Systems, 9(2):171-180, 2000 .

14. J.-M. Lehn. Supramolecular Chemistry: Concepts and Perspectives. Wiley, 1995.

15. R. C. Mucic, J. J. Storhoff, C. A. Mirkin, and R. L. Letsinger. DNA-directed synthesis of binary nanoparticle network materials. Journal of the American Chemical Society, 120:12674-12675, 1998.

16. V. N. Paunov, P. A. Kralchevsky, N. D. Denkov, and K. Nagayama. Lateral capillary forces between floating submillimeter particles. Journal of Colloid and Interface Science, 157:100-112, 1993.

17. H. M. Princen. Wetability and contact angles. In E. Matijević, editor, Surface and Colloid Science, volume 2, pages 1-153. Wiley, 1969.

18. H. Reif and H. Wang. Social potential fields: A distributed behavioral control for autonomous robots. In Proceedings of the 1994 Workshop on the Algorithmic Foundations of Robotics. A.K.Peters, Boston, MA, 1995.

19. E. Rimon and D.E. Koditschek. Exact robot navigation using artificial potential fields. IEEE Transactions on Robotics and Automation, 8(5):501-518, October 1992.

20. W. K. Rothemund. Using lateral capillary forces to compute by self-assembly. PNAS, 97(3):984-989, 2000.

21. K. Saitou. Conformational switching in self-assembling mechanical systems. IEEE Transactions on Robotics and Automation, 15(3):510-520, June 1999.

22. Y. Wang, X. Cheng, Y. Hanein, B. D. Ratner, and K. F. Böhringer. Protein patterning with programmable surface chemistry chips. In Proceedings of the Sixth International Symposium on Micro Total Analysis System ( $\mu$ TAS), Nara, Japan, November 2002.

23. E. Winfree. Algorithmic self-assembly of DNA: Theoretical motivations and 2D assembly experiments. Journal of Biomolecular Structure and Dynamics, 11(2):263-270, May 2000.

24. H.-J.J. Yeh and J.S. Smith. Fluidic assembly for the integration of GaAs light-emitting diodes on $\mathrm{Si}$ substrates. IEEE Photonics Technology Letters, 6:706-708, 1994. 